\title{
Factors Associated with In-hospital Mortality in Patients with Acute Coronary Syndrome
}

\author{
Andreea Barcan, Istvan Kovacs, Ciprian Blendea, Marius Orzan, Monica Chitu
}

University of Medicine and Pharmacy, Tîrgu Mureș, Romania

\section{ABSTRACT}

Introduction: The recent development of large networks dedicated to ST-segment elevation myocardial infarction (STEMI) led to a significant increase in the number of primary percutaneous interventions ( $\mathrm{p}-\mathrm{PCI}$ ) parallel with mortality reduction in Acute Coronary Syndrome (ACS). The number of non ST segment elevation myocardial infarction (NSTEMI) is increasing and the highest mortality rates are encountered in patients with cardiogenic shock and/or out of hospital cardiac arrest associated to ACS. The aim of this study was to identify the factors associated with a higher mortality rate in a global population with acute coronary syndromes presented in the emergency department of a county clinical hospital which serves as a regional center for a STEMI network.

Material and method: This is a retrospective study including 684 patients with acute coronary syndrome admitted in the Clinic of Cardiology from the County Clinical Emergency Hospital Tîrgu Mureș in 2014. In all the cases, the factors that correlated with in hospital mortality were identified and analyzed.

Results: The incidence of arterial hypertension was significantly higher in patients admitted with unstable angina (75.0\%) and STEMI cases with less than 12 hours onset of symptomatology $(68.1 \%)$, while impaired renal function correlated with the presence of NSTEMI (66.6\%). The presence of a multivessel disease was significantly correlated with cardiogenic shock. The localisation of the culprit lesion in the left anterior descending artery (LAD) significantly correlated with the development of cardiogenic shock, LAD culprit lesions being present in $44.4 \%$ of CS cases as compared with $21.7 \%$ of noCS cases in STEMI patients. In NSTEMI patients, the localisation of the culprit lesion in the left main artery (LM) significantly correlated with the development of cardiogenic shock, culprit lesions in the left main being present in $47.0 \%$ of CS cases as compared with $28.5 \%$ of noCS cases in STEMI patients.

Conclusion: Patients presenting with out-of-hospital resuscitated cardiac arrest due to Acute Myocardial Infarction associate higher in-hospital mortality rates. In-hospital mortality seems to be highly correlated with the female gender, STEMI myocardial infarction and the presence of multivascular lesions.

Keywords: acute coronary syndrome, in-hospital mortality, acute myocardial infarction

\section{ARTICLE HISTORY}

Received: 5 September, 2015

Accepted: 10 November, 2015

\section{CORRESPONDENCE}

\section{Istvan Kovacs}

University of Medicine and Pharmacy of Tîrgu Mureș, Clinic of Cardiology

50 Gheorghe Marinescu St 540136 Tîrgu Mureș, Romania Tel/Fax: +40-265-211595

Email: kov_istvan@yahoo.com 


\section{INTRODUCTION}

The recent development of large networks dedicated to ST-segment elevation myocardial infarction (STEMI) led to a significant increase in the number of primary percutaneous interventions (p-PCI), which became largely accessible to STEMI population [1]. However, mortality in acute coronary syndromes remains high, especially in cases with associated cardiogenic shock or cardiac arrest $[2,3]$. Recent studies demonstrated that a younger age in STEMI cases is associated with a better prognosis. In nonST elevation myocardial infarction (NSTEMI) quantitative assessment of risk based on risk scores is superior to the clinical assessment in providing an objective estimation of the risk associated with the acute event [4]. The GRACE risk score provides the most accurate stratification of risk both at admission in the hospital and at discharge [1].

The assessment of acute risk is helpful in guiding the initial evaluation, for selection of the site of care (coronary or intensive care unit, intermediate care unit, or regular unit), therapy and timing of coronary angiography. The risk is highest at the time of presentation and may remain elevated for several days, although rapidly declining over time, depending on clinical presentation, comorbidities, coronary anatomy and revascularization.

Factors with the highest independent significance in terms of mortality prediction include age, baseline serum creatinine, systolic blood pressure, troponin levels, cardiogenic shock at presentation, dynamic ST-segment changes, heart rate, and the co-existence of severe peripheral arterial disease [5,6].

Increasing clinical experience recommends that resuscitated cardiac arrest patients without an obvious cardiac etiology should undergo emergency coronary angiography and, where indicated, percutaneous coronary intervention [1].

In addition to short-term risk factors, a number of conditions are associated with long-term risk, including a complicated clinical course, left ventricular systolic dysfunction, severity of coronary artery disease, revascularization status, evidence of residual ischaemia on non-invasive testing or non-cardiac co-morbidities. The presence of arterial hypertension or diabetes has been demonstrated to increase in hospital mortality rates. Angiographic findings such as the presence of multivessel lesions or left main involvement have also been demonstrated to be associated with a higher risk in ACS patients. In contemporary registries, the rates of death, myocardial infarction and recurrent acute coronary syndrome at 1 year are around $10 \%$ [7]. While early events are related to ruptured coronary plaques and associated thrombosis, the majority of later events may be the result of coronary and systemic atherosclerosis progression.

Multiple biomarkers have been associated with mortality in acute coronary syndromes (ACS), several of them with additive prognostic value to cardiac troponin [8]. Serum creatinine and estimated glomerular filtration rate (eGFR) should also be determined in all patients with ACS, as they are directly correlated to survival and short term prognosis.

The aim of this study was to identify the factors associated with a higher mortality rate in a global population with acute coronary syndromes presented in the emergency department of a county clinical hospital which serves as a regional center for a STEMI network.

\section{MATERIAL AND METHODS}

This is a retrospective study including 684 patients with acute coronary syndrome admitted in the Clinic of Cardiology from the County Clinical Emergency Hospital Tirgu Mures in 2014. The study was approved by the ethics committee of the County Clinical Emergency Hospital Tirgu Mures and by the ethics committee of the University of Medicine and Pharmacy of Tirgu Mures, Romania.

The acute coronary syndrome was in 413 cases an acute myocardial infarction with ST-segment elevation presenting at less than 12 hours from onset of symptoms (60.3\%), in 47 cases an acute myocardial infarctions with ST-segment elevation presenting at more than 12 hours from onset of symptoms (8.3\%), in 123 cases an acute myocardial infarctions without ST-segment elevation (17.9\%) and in 91 patients an unstable angina (13.3\%) (Table 1). From the 684 patients admitted with acute coronary syndrome, 78 died, with a global rate of mortality of $11 \%$. From the 78 deceased patients 44 were women $(56.4 \%)$, and 34 were men $(43.5 \%)$.

In all the cases, the factors that correlated with in hospital mortality were identified and analyzed.

Statistical analysis was performed using the JMP 10 statistical software (SAS Institute Inc., Cary, North Caro-

TABLE 1. Type of Acute Coronary Syndrome

\begin{tabular}{lcc}
\hline ACS type & $\mathbf{N}=\mathbf{6 8 4}$ & $\mathbf{\%}$ \\
\hline STEMI $<24 \mathrm{~h}$ & 413 & $60.3 \%$ \\
STEMI $>24 \mathrm{~h}$ & 57 & $8.3 \%$ \\
NSTEMI & 123 & $17.9 \%$ \\
UA & 91 & $13.3 \%$ \\
\hline
\end{tabular}




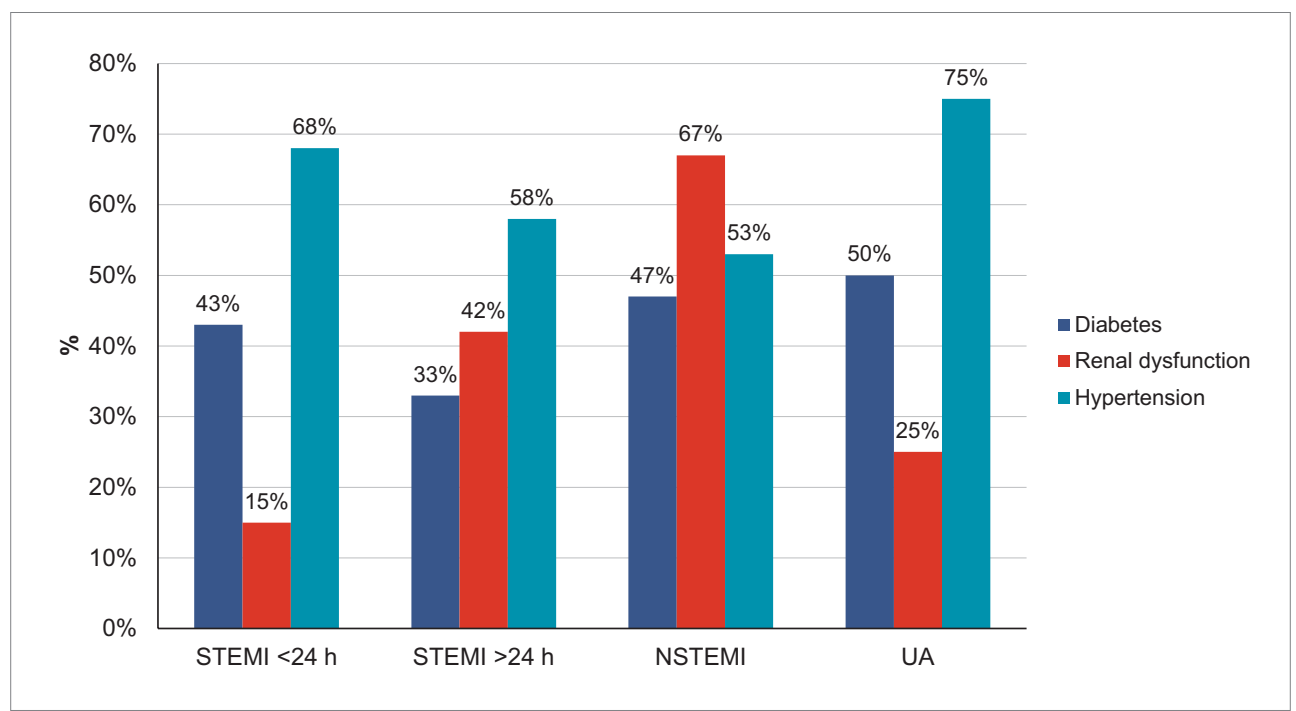

FIGURE 1. Risk factors associated with ACS

lina). Statistical significance was considered for a $\mathrm{p}$ value $<0.05$, and all $\mathrm{p}$ values were 2 -sided.

\section{RESULTS}

Factors associated with mortality in the study lot included age, gender, troponin levels, necessity of intra-aortic balloon pump insertion, dynamic ST-segment changes, multivascular disease, out of hospital resuscitation and the presence of impaired renal function, arterial hypertension, diabetes and cardiogenic shock at presentation (Table 2).

From the patients who died in the hospital, 58 patients were initially successfully resuscitated, 28 in prehospital, and 30 patients during hospitalization. The place of resuscitation did not present any statistically significant correlation with in hospital mortality.

Coronarography was performed in 62 cases $(79.4 \%)$, and was followed by PCI in 61 patients $(78.2 \%)$. The presence of multivessel disease, encountered in 44 patients (56.4\%) turned out to represent an important predictor for in hospital mortality.

A significant correlation was found between the presence of several risk factors and in hospital mortality. The incidence of arterial hypertension was significantly higher in patients admitted with unstable angina (75.0\%) and acute myocardial infarction with ST elevation with less than 12 hours onset of symptomatology $(68.1 \%)$, while impaired renal function correlated with the presence of acute myocardial infarction without ST elevation (66.6\%). Diabetes was present in 50\% of the cases admitted with unstable angina (Figure 1).
In patients who underwent PCI, the correlation between the time from first medical contact (FMC) to baloon and in hospital mortality was analyzed. FMC to baloon time was similar in patients with out of hospital cardiac arrest and in those with in-hospital cardiac arrest, indicating that the ischemia time was almost equal in both groups.

From the 78 patients presenting with cardiogenic shock, 42 patients $(53.8 \%)$ needed insertion of IABP. The

TABLE 2. Characteristics of deceased ACS patients

\begin{tabular}{|c|c|c|}
\hline Characteristics & $\mathrm{N}=78$ & $\%$ \\
\hline Mean age (years) & $70.82 \pm 10.2$ & \\
\hline Male & 34 & $43.5 \%$ \\
\hline Female & 44 & $56.4 \%$ \\
\hline Diabetes & 29 & $37.1 \%$ \\
\hline Renal dysfunction & 23 & $29.4 \%$ \\
\hline Hypertension & 43 & $55.1 \%$ \\
\hline \multicolumn{3}{|l|}{ Acute coronary syndrome } \\
\hline STEMI $<24$ h & 47 & $60.2 \%$ \\
\hline STEMI $>24 \mathrm{~h}$ & 12 & $15.3 \%$ \\
\hline NSTEMI & 15 & $19.2 \%$ \\
\hline $\mathrm{UA}$ & 4 & $5.1 \%$ \\
\hline Cardiogenic shock at admission & 42 & $53.8 \%$ \\
\hline Prehospital resuscitation & 28 & $35.9 \%$ \\
\hline In hospital resuscitation & 30 & $38.4 \%$ \\
\hline PCI & 61 & $78.2 \%$ \\
\hline No PCI & 17 & $21.79 \%$ \\
\hline Single-vessel disease & 18 & $23.1 \%$ \\
\hline Multivessel disease & 44 & $56.4 \%$ \\
\hline IABP & 7 & $8.9 \%$ \\
\hline
\end{tabular}




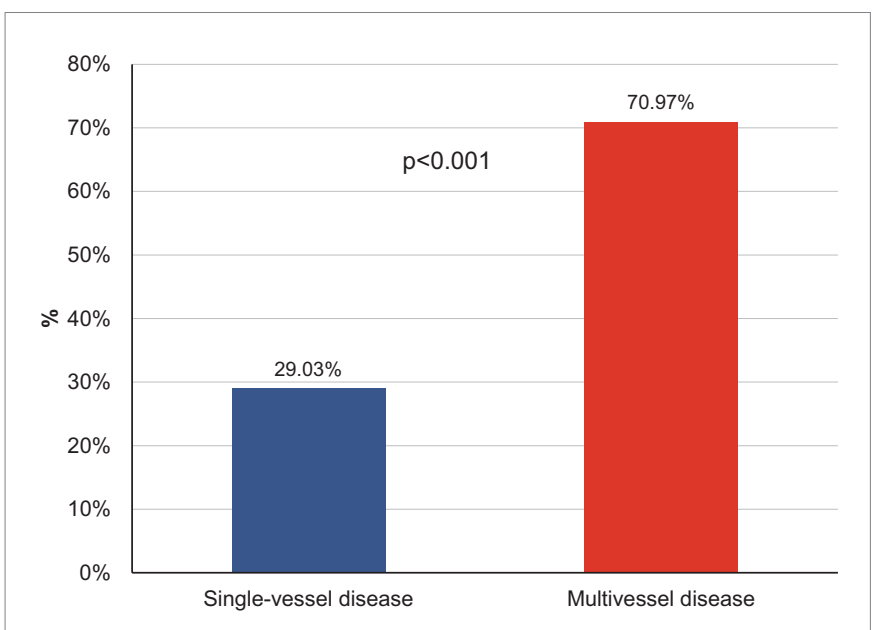

FIGURE 2. Association between multivessel disease and cardiogenic shock

presence of a multivessel disease was significantly correlated with cardiogenic shock (Figure 2).

Patients with cardiogenic shock (CS) who were admitted in intensive care unit following PCI presented a significantly higher risk for complications as compared with patients without cardiogenic shock (Table 3). At the same time, the mean age of patients who required a longer than 24 hours stay in the intensive care unit was significantly higher than the mean age of patients who spent less than 24 hours in the intensive care $(73.0 \pm 8.5$ vs $64.4 \pm 11.2$, p $=0.007$ ) (Figure 3).

Analysis of the association between the location of the coronary lesions and the occurence of cardiogenic shock in STEMI patients indicated that the localisation of the cul-

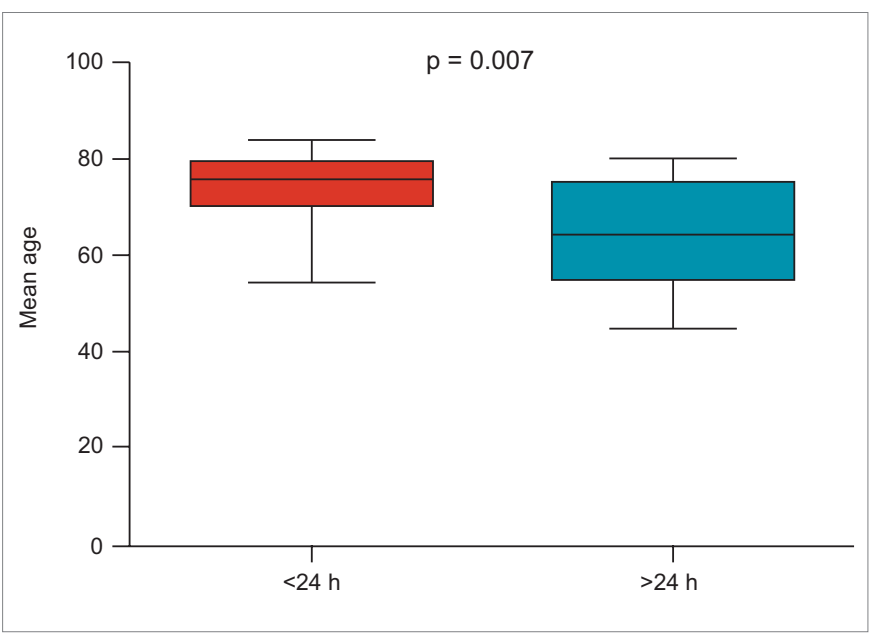

FIGURE 3. Mean age of patients who required more than 24 hours versus those who required less than 24 hours stay in the intensive care unit.

prit lesion in the left anterior descending artery (LAD) significantly correlated with the development of cardiogenic shock, LAD culprit lesions being present in $44.4 \%$ of CS cases as compared with $21.7 \%$ of noCS cases in STEMI patients (Figure 4). In NSTEMI patients, the localisation of the culprit lesion in the left main artery (LM) significantly correlated with the development of cardiogenic shock, culprit lesions in the left main being present in $47.0 \%$ of CS cases as compared with $28.5 \%$ of noCS cases in STEMI patients (Figure 5).

Elevated troponin at admission did not present any significant correlation with the presence of cardiogenic shock.

The presence of severe arrythmia was encountered in $68.2 \%$ of cases in patients with multivessel disease, and

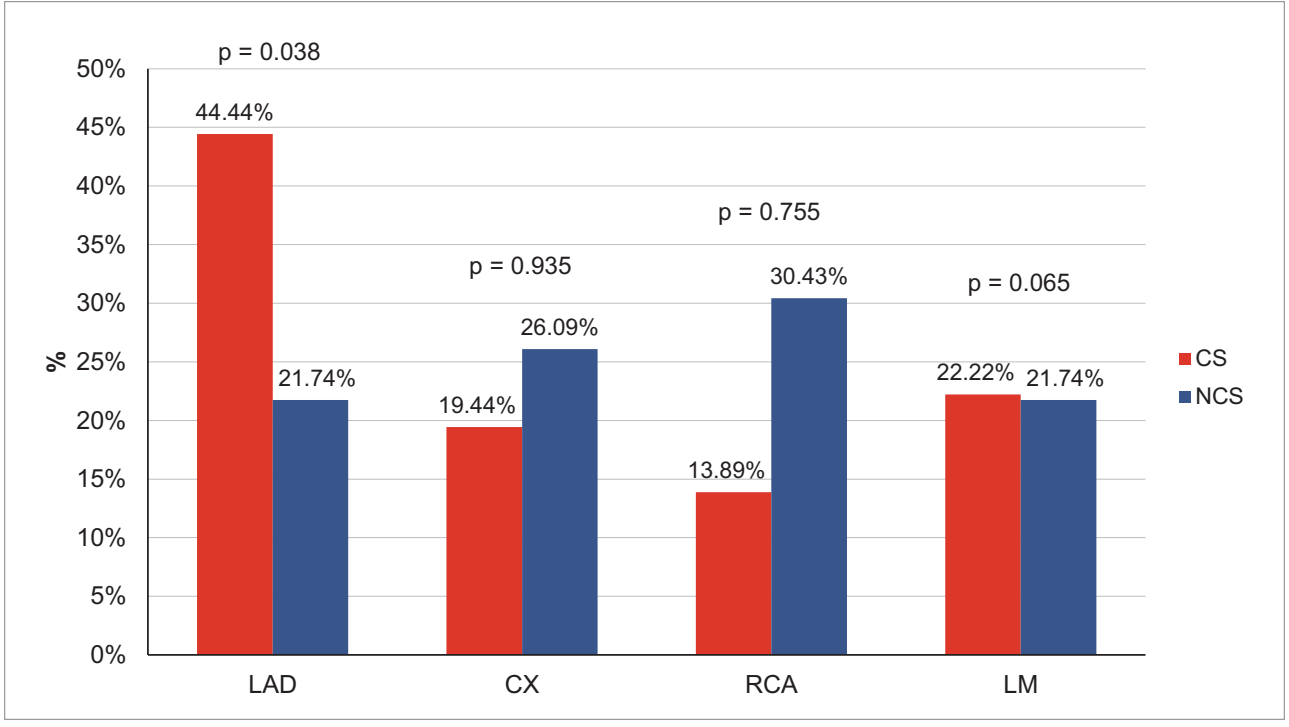

FIGURE 4. Association between location of culprit lesion and cardiogenic shock in STEMI patients. LAD - left anterior descending artery, CX - circumflex artery, RCA - right coronary artery, LM - left main 


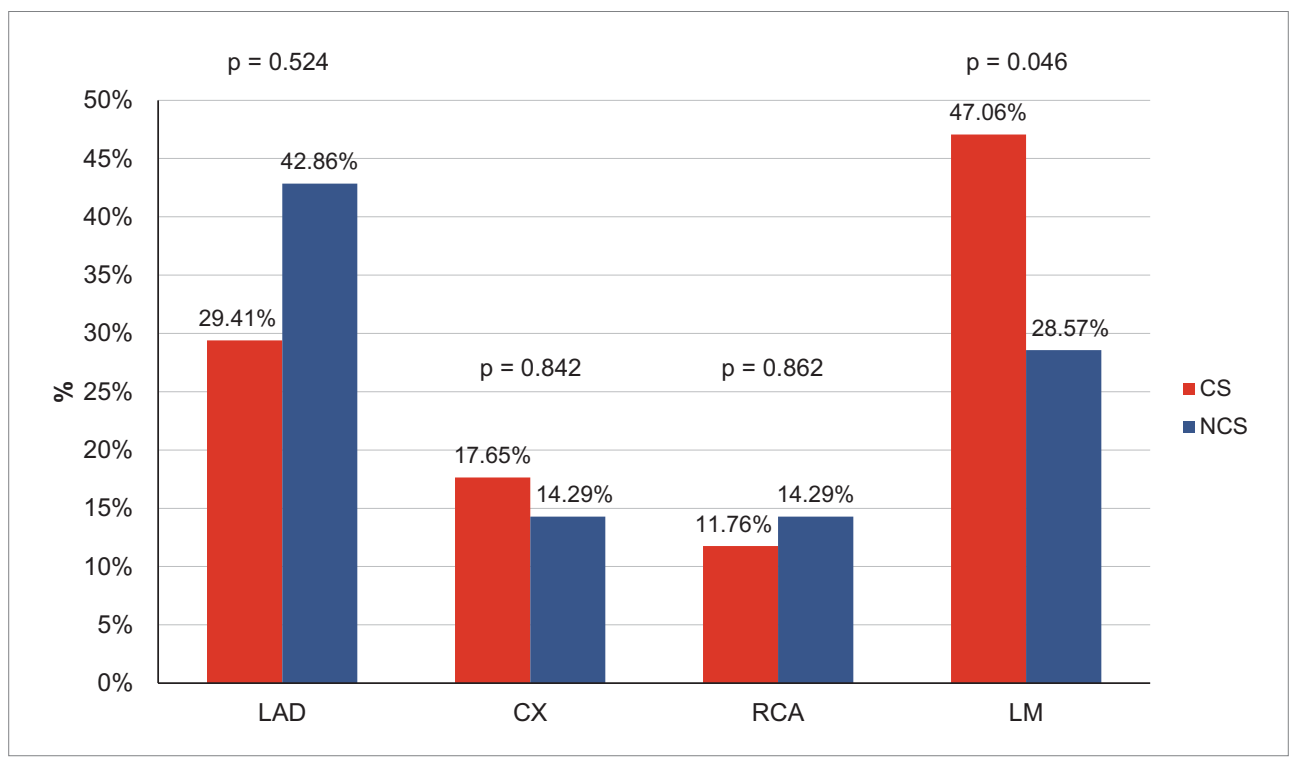

FIGURE 5. Association between location of culprit lesion and cardiogenic shock in NSTEMI patients. LAD - left anterior descending artery, CX - circumflex artery, RCA - right coronary artery, LM - left main

only in $31.8 \%$ of cases in patients with univessel disease, indicating that the arrythmogenic substratum in these cases could be represented by extensive zones of ischaemia determined by the multivessel disease.

\section{DISCUSSIONS}

The ACS-related in-hospital mortality shows a decreasing tendency nowadays, mainly as a result of a better prehospital care and superior network organization, leading to a higher number of primary PCI procedures. According to data published in the literature, the mortality in NSTEMI is slightly higher than the global mortality of ACSs $[7,8]$. Similarly, our study showed a higher mortality in NSTEMI cases, mainly explained by a higher mean age of this subset of patients and a higher incidence of left main culprit lesions, especially in cases associated with cardiogenic shock.

In a recent study, the mean age of ACS patients presenting with cardiogenic shock, cardiac arrest and out of hospital resuscitation was 56.1 years for those with single vessel disease and 67.2 for those with multivessel disease [9]. In our study group, the mean age of ACS patients was 70.8 years, this difference being explained by the significantly higher number of patients with multivessel disease in the study lot.

In STEMI patients the male gender represents a predisposing factor for cardiogenic shock, however in NSTEMI cases the percentage of female gender with cardiogenic shock is increasing. Published data indicates significantly higher in-hospital mortality in women with cardiogenic shock than in men with CS (21.5\% vs $8.6 \%$ ) [10]. This was also noted in the present study, which indicated a higher incidence of female gender as compared to male in the deceased population ( $56.4 \%$ vs $43.5 \%$ ).

The major cardiovascular risk factors (hypertension, diabetes, renal dysfunction) are very common in ACS.

TABLE 3. Complication rates in patients with CS versus those with no CS

\begin{tabular}{|c|c|c|c|}
\hline Complications & $\operatorname{CS}(n=42)$ & $\mathrm{nCS}(\mathrm{n}=36)$ & $\%$ \\
\hline Anemia & 0 & 1 & $1(1.3 \%)$ \\
\hline Haemorrhagic stroke & 0 & 1 & $1(1.3 \%)$ \\
\hline Pneumonia & 2 & 0 & $2(2.6 \%)$ \\
\hline Acute colecistitis & 0 & 1 & $1(1.3 \%)$ \\
\hline $\begin{array}{l}\text { Upper gastrointenstinal } \\
\text { bleeding }\end{array}$ & 1 & 2 & $3(3.8 \%)$ \\
\hline Mezenteric infarction & 0 & 1 & $1(1.3 \%)$ \\
\hline Acute Renal Failure & 0 & 1 & $1(1.3 \%)$ \\
\hline Acute limb ischaemia & 0 & 1 & $1(1.3 \%)$ \\
\hline MSOF & 0 & 1 & $1(1.3 \%)$ \\
\hline Cardiac rupture & 2 & 3 & $5(6.4 \%)$ \\
\hline Hepatorenal syndrome & 0 & 2 & $2(2.6 \%)$ \\
\hline Sepsis & 4 & 6 & $10(12.8 \%)$ \\
\hline Haemorrhagic shock & 0 & 1 & $1(1.3 \%)$ \\
\hline Tamponade & 0 & 1 & $1(1.3 \%)$ \\
\hline \multirow[t]{2}{*}{ Acute in-stent thrombosis } & 0 & 1 & $1(1.3 \%)$ \\
\hline & $42(53.8 \%)$ & $36(46.2 \%)$ & 78 \\
\hline
\end{tabular}


Diabetes is an independent predictor for complications in patients undergoing a revascularization procedure [11]. Diabetes and renal dysfunction (diabetic or hypertensive nephropathy) can lead to major complications such as bleeding or depressed immunity status resulting in a higher rate of infections [11]. In our study diabetes was present in $37.2 \%$ of cases, renal dysfunction in $29.5 \%$ of cases and hypertension in $55.1 \%$ of patients, with a higher incidence of renal dysfunction in NSTEMI patients. In this subgroup the multivessel coronary diseases was more freqvent, longer PCI procedures were necessary, with higher amounts of contrast medium administration. All these could represent a possible explanation for the higher complication rate and higher mortality recorded in this group.

Multivessel disease occurs in up to $87 \%$ of patients with CS and is accompanied by a high mortality rate [9]. In our study we noted a significantly higher percentage of patients admitted with multivessel diseases as compared with single-vessel disease [70.9\% vs $29.03 \%$ ]. In complex cases of multivessel disease the revascularization procedure is longer due to the need of complete revascularization, however in many cases such a complex strategy cannot be applied due to the co-existence of renal dysfunction [12].

It is well-known that in STMI cases early revascularization therapy leads to superior rates of short and longterm survival. However, the success rate of the revascularization procedure is only $75 \%$ in patients admitted in cardiogenic shock and these data are similar in patients with ACS and out of hospital cardiac arrest [13]. In our study, we noted a $78.2 \%$ succes rate of PCI in the study lot, $74.35 \%$ of these patients presentin cardiac arrest prior to revascularization.

Data from literature shows a high rate of associations between left main stenosis and multivessel diseases in patients with ACS and CS, however there are only few data published regarding the association between left main stenosis and out of hospital cardiac arrest or cardiogenic shock in NSTEMI patients [14]. The present study showed a significantly higher rate of LAD lesions in patients with STEMI and CS (44\%). Also, the presence of a significant left main disease was noted in $47 \%$ of cases with NSTEMI and CS. The factors that presented a higher association with death in our study were advanced age, female gender, out of hospital cardiac arrest, cardiogenic shock and location of the culprit lesion in the left anterior descending artery in STEMI cases, respectively the presence of multivessel disease with involvement of the left main in NSTEMI patients.

\section{CONCLUSIONS}

Patients presenting with out-of-hospital resuscitated cardiac arrest due to Acute Myocardial Infarction associate higher in-hospital death rates. Revascularisation of culprit lesion and optimal intensive care are the most important strategies in reducing the mortality rate in these complex cases.

In-hospital mortality seems to be highly correlated with the female gender, STEMI myocardial infarction and the presence of multivessel disease. The most frequent arrythmia causing the cardiac arrest was predominantly ventricular, and the arrythmic event correlated with the presence of multivessel disease.

\section{REFERENCES}

1. Windecker S, Kolh P, Fernando A et al. 2014 ESC/EACTS Guidelines on myocardial revascularization. European, The Task Force on Myocardial Revascularization of the Society of Cardiology (ESC) and the European Association for CardioThoracic Surgery (EACTS). Eur Heart J. 2014;35:2541-2619.

2. Garot $\mathrm{P}$, Lefevre $\mathrm{T}$, Eltchaninoff $\mathrm{H}$, et al. Six-Month Outcome of Emergency Percutaneous Coronary Intervention in Resuscitated Patients After Cardiac Arrest Complicating STElevation Myocardial Infarction. Circulation. 2007;115:13541362.

3. Hasselqvist-Ax I, Riva G, Herlitz J et al. Early Cardiopulmonary Resuscitation in Out-of-Hospital Cardiac Arrest. New Eng J Med. 2015;372:2307-2315.

4. Roffi M, Patrono C, Collet JP, et al. 2015 ESC Guidelines for the management of acute coronary syndromes in patients presenting without persistent ST-segment elevation: Task Force for the Management of Acute Coronary Syndromes in Patients Presenting without Persistent ST-Segment Elevation of the European Society of Cardiology (ESC). Eur Heart J. 2015; pii: ehv320. [Epub ahead of print]

5. Collinson J, Flather MD, Fox KA, et al. Clinical outcomes, risk stratification and practice patterns of unstable angina and myocardial infarction without ST elevation: prospective registry of acute ischaemic syndromes in the UK (PRAIS-UK). Eur Heart J. 2000;21:1450-1457.

6. Ruiz Bailén M, Rucabado-Aguilar L, Castillo-Rivera AM, et al. Cardiogenic shock in acute coronary syndromes in the Spanish population. Med Sci Monit. 2008;14:PH46-57.

7. McManus DD, Gore J, Yarzebski J, et al. Recent trends in the incidence, treatment, and outcomes of patients with STEMI and NSTEMI. Am J Med. 2011;124:40-47.

8. Montalescot G, Dallongeville J, van Belle E, et al. STEMI and NSTEMI: Are they so different? 1 year outcomes in acute myocardial infarction as defined by the esc/acc definition (the opera registry). Eur Heart J. 2007;28:1409-1417.

9. Mylotte D, Morice MC, Eltchaninoff $\mathrm{H}$, et al. Primary percutaneous coronary intervention in patients with acute myocardial infarction, resuscitated cardiac arrest, and cardiogenic shock: the role of primary multivessel revascularization. J Am Coll Cardiol Intv. 2013;6:115-125. 
10. Alonso J, Bueno H, Bardají, A et al. Influence of sex on acute coronary syndrome mortality and treatment in Spain. Rev Esp Cardiol Supl. 2008; 8:8D-22D.

11. Fuster V, Farkouh ME. Coronary Syndromes and Diabetes Mellitus. Circulation. 2008;118:1607-1608.

12. Park JS, Cha KS, Lee DS, et al. Culprit or multivessel revascularisation in ST-elevation myocardial infarction with cardiogenic shock. Heart. 2015;101:1225-1232.
13. Awad HH, Anderson FA Jr, Gore JM et al. Cardiogenic shock complicating acute coronary syndromes: Insights from the Global Registry of Acute Coronary Events. Am Heart J. 2012;163:963-971.

14. Almudarra SS, Gale CP, Baxter PD et al. Comparative Outcomes After Unprotected Left Main Stem Percutaneous Coronary Intervention. JACC Cardiovasc Interv. 2014;7:717-730. 